\title{
GPT3Mix: Leveraging Large-scale Language Models for Text Augmentation
}

\author{
Kang Min Yoo ${ }^{1,2}$, Dongju Park ${ }^{2}$, Jaewook Kang ${ }^{2}$, \\ Sang-Woo Lee ${ }^{1,2}$, and Woomyeong Park ${ }^{2}$ \\ ${ }^{1}$ NAVER AI Lab \\ ${ }^{2}$ NAVER Clova AI \\ \{kangmin.yoo, dongju.park, jaewook.kang\}@navercorp.com \\ \{sang.woo.lee, max.park\}@navercorp.com
}

\begin{abstract}
Large-scale language models such as GPT3 are excellent few-shot learners, allowing them to be controlled via natural text prompts. Recent studies report that prompt-based direct classification eliminates the need for finetuning but lacks data and inference scalability. This paper proposes a novel data augmentation technique that leverages large-scale language models to generate realistic text samples from a mixture of real samples. We also propose utilizing soft-labels predicted by the language models, effectively distilling knowledge from the large-scale language models and creating textual perturbations simultaneously. We perform data augmentation experiments on diverse classification tasks and show that our method hugely outperforms existing text augmentation methods. We also conduct experiments on our newly proposed benchmark to show that the augmentation effect is not only attributed to memorization. Further ablation studies and a qualitative analysis provide more insights into our approach.
\end{abstract}

\section{Introduction}

In the seminal work by Brown et al. (2020), a largescale language model, specifically GPT-3, has been shown to achieve superior performance on zeroshot and few-shot learning tasks by prompt-based in-context learning. In-context learning utilizes a prompt, which usually consists of a task description and few examples, to solve unseen tasks without the hefty price of fine-tuning. Recognizing the potential research applications of in-context learning and prompt-based control, a part of the NLP community has shifted its focus on understanding and devising advanced methods for optimizing promptbased approaches (Schick and Schütze, 2020a; Shin et al., 2020; Zhao et al., 2021; Reynolds and McDonell, 2021).

However, these prompt-based approaches with inference on a large-scale language model suffer

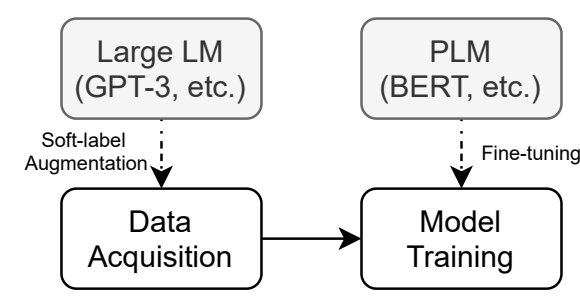

Figure 1: A conceptual diagram of text augmentation using large-scale language models.

from several drawbacks. First, the number of incontext training examples is hard limited by the maximum prompt length enabled by the inherent language model architecture. Second, promptbased approaches require online inference on the expensive large-scale language models. The inference may not be scalable in real-world use cases, because it is slow and incurs huge memory overhead. Lastly, the prompt-based approaches do away with conventional machine learning techniques, making it mostly incompatible with existing established fine-tuning methods.

To overcome such limitations, we propose a more practical solution to utilize large-scale language models for downstream NLP tasks. In our proposed framework, as depicted in Figure 1 , large-scale language models are not used as the pre-trained model for further domain-adaptive fine-tuning nor the backbone for prompt-based incontext learning but for imbuing the original training set with synthetic text data.

We propose GPT3Mix, a method for generating synthetic but hyper-realistic text samples from a mixture of real samples utilizing large-scale language models such as GPT- $3^{1}$. GPT3Mix extracts few sample sentences from the task-specific training data, embed these samples in the prompt, and

\footnotetext{
${ }^{1}$ Despite what the name suggests, we can apply GPT3Mix to any large-scale autoregressive language models.
} 
generates an augmented mixed sentence influenced by the sample sentences. GPT3Mix uses softlabels predicted by the large-scale language model to transfer knowledge of probability as in knowledge distillation (Hinton et al., 2015). In short, our method achieves both (1) data augmentation via generating synthetic examples inspired by existing data samples and (2) knowledge distillation by training smaller classification models using softlabels predicted by the large language model.

Our approach takes inspiration from the mixbased data augmentation methods in the vision domain (Zhang et al., 2017). Several mix-based data augmentation methods are suggested for NLP models. One of the notable methods is MixText (Chen et al., 2020), in which BERT is used to generate novel augmentation samples from interpolated embedding spaces. However, despite its great success in the vision domain, deep-mixing text augmentation methods have seen limited effectiveness in realworld cases due to the difficulty of interpolating language from latent spaces (Bowman et al., 2016). Synthetic language interpolated from a model's hidden space such as the word embedding space of BERT may introduce noise, outweighing the benefit of novel sample discovery and causing deterioration in the training data distribution. Our work exploits the generative power of large-scale language models like GPT-3 to generate high-quality mixed samples from in-context examples.

We perform various data augmentation experiments on diverse classification tasks to verify our hypotheses and analyze our methodology. As language models are partly pretrained on web-crawled corpora, some benchmarks such as the movie review classification tasks may have been "seen" by the language models. To eliminate the possibility of pretraining memorization, we propose a new task RT2O where we collected online movie reviews posted after the known data preparation date of GPT-3. Experimental results with the newly proposed benchmark RT20 show that the benefit of our method is not attributed to memorization but mix-based text synthesis. We will release the benchmark soon.

The contribution of our work is summarized as follows. ${ }^{2}$

1. We suggest employing prompt-based data augmentation using large-scale language mod-

\footnotetext{
${ }^{2}$ The code to reproduce our results is available at https://github.com/naver-ai/hypermix.
}

els on top of the existing PLM fine-tuning paradigm to exploit the best of both worlds.

2. We propose GPT3Mix, a simple but effective text augmentation technique, that elicits knowledge and linguistic capability possessed by large-scale language models.

3. Our detailed analysis helps to understand the mechanism behind prompt-powered data augmentation, giving us insights into the generation and augmentation behavior.

4. Our newly proposed RT20 task enables controlled experimentation on language models pretrained prior to a certain date, eliminating the possibility of memorization.

\section{Related Work}

Knowledge Distillation Knowledge distillation (Phuong and Lampert, 2019) is a technique that trains a smaller student classifier on the outputs of a larger teacher classifier. Knowledge distillation for language models in the context of model compression has been well-studied in the literature. There have been various distilled models and distillation methods proposed for pre-trained language models (Sanh et al., 2019; Tang et al., 2019). By utilizing soft-labels predicted by the large-scale language model, our approach helps to transfer knowledge to the downstream classifiers.

Text Augmentation Text augmentation refers to methods for perturbing the linguistic space without altering class labels to improve the robustness and generalizability of the downstream models. Data augmentation has been studied extensively in the NLP scene. Text augmentation in the current literature comes with two flavors: shallow and deep augmentation. The shallow data augmentation techniques inject locally plausible small noises into the linguistic space (words or phrases), in the hopes that the perturbations produce linguistically acceptable samples while maintaining label consistency. Two examples are EDA (Wei and Zou, 2019) and synonym replacement (Zhang et al., 2016).

Another class of augmentation techniques employs external language models to improve global coherence and consistency. The back-translation approach exploits semantic consistency in translation language pairs to generate novel paraphrases (Fadaee et al., 2017). In the more recent line of work, pre-trained language models, such as BERT 
(Devlin et al., 2019) or the sequence-to-sequence variant BART (Lewis et al., 2020), are used to obtain more diverse and linguistically correct augmentation samples. For example, BART has been proven to be effective in populating text samples for data-scarce labels (Kumar et al., 2020). Ng et al. (2020) proposed using masked language models as a denoising autoencoder to generate synthetic texts. Some other researchers have taken the direction of perturbing the latent spaces, optionally by introducing variational inference in the architecture (Xia et al., 2020b,a; Hou et al., 2018; Yoo et al., 2019).

On the other hand, inspired by the mix-up technique (Zhang et al., 2017) proposed for the vision domain, there have also been works to mix existing text samples to produce realistic augmentation texts based on statistical methods (Guo et al., 2020; Sun et al., 2020; Chen et al., 2020). Furthermore, pseudo-labeling, the act of annotating unlabeled data with model predictions (Lee et al., 2013; Reed et al., 2014), has been actively used in semi-supervised learning settings (Chen et al., 2020; Xie et al., 2020; Berthelot et al., 2019).

Large-scale Language Models Pre-trained transformer-based language models (Devlin et al., 2019; Lewis et al., 2020) have initiated a new paradigm in the NLP scene, changing the way we design NLP pipelines. With the recent development of mega-scale language models (Shoeybi et al., 2019; Brown et al., 2020), we are witnessing another shift in the paradigm, namely prompt-based NLP. These large language models are essentially few-shot learners, allowing them to be controlled through natural text. There has been a steep rise in the community's interest to better understand the prompt-based mechanisms (Reynolds and McDonell, 2021; Schick and Schütze, 2020a; Shin et al., 2020; Jiang et al., 2020; Zhao et al., 2021). Our work relies on the previous findings on prompt-based manipulation.

To the best of our knowledge, this work is the first to propose using the prompt-based approach to generate synthetic samples from large-scale language models for the purpose of text augmentation.

\section{GPT3Mix}

Mixup (Zhang et al., 2017) is a simple learning technique that has been shown to be effective in preventing memorization and improving generalizability for the vision domain. The technique has been very effective on image data, but it has been harder to establish a standard approach for texts due to the inherent sparse nature of linguistic distributions, which attributes to the challenges of identifying adversarial text examples (Li et al., 2017). Inspired by the technique, we propose GPT3Mix as a powerful yet simple method to generate highly fluent synthetic samples based on a data distribution.

The proposed method (Figure 2) consists of three steps: (1) selecting examples from the dataset, (2) constructing a GPT3Mix prompt from the selected examples and meta-information about the dataset, and finally (3) extracting augmentation from the language model generation. This section provides details about each step as follows.

Example Selection For simplicity, we confine the downstream task to text classification tasks. Given a classification task $\mathcal{T}$, the training dataset $\mathcal{D}$ is a set of text $\mathbf{x}$ and associated label $y$ pairs: $\mathcal{D}=\left\{\left(\mathbf{x}_{i}, y_{i}\right) \mid 1 \leq i \leq N\right\}$.

We randomly choose $k$ examples from $\mathcal{D}$ to be anchors. Large-scale language models are known to be highly sensitive to the choice and the order of examples in the prompt (Reynolds and McDonell, 2021; Zhao et al., 2021). We conjecture that by carefully choosing the examples, we are able to control the generated augmentation samples from the language model. We conduct qualitative analysis on the augmentation samples to confirm our hypothesis (§4.4.5).

In our implementation, we simply used uniform distribution to choose $k$ examples: $p_{s}(i)=1 / N$. Otherwise stated, most experiments are carried out by setting $k=2$ to simulate Mix-up. As found in our ablation studies (\$4.4.1), $k=2$ provides a good trade-off between cost and performance.

Prompt Construction Given a set of prompt examples $\mathcal{D}_{e}=\left\{\left(\mathbf{x}_{i}, y_{i}\right) \mid 1 \leq i \leq k\right\}$ sampled from $\mathcal{D}$, we formulate the prompt as follows.

A GPT3Mix prompt consists of a description header, an enumeration of text-label pairs of $\mathcal{D}_{e}$, and the augmentation prefix. An example of the prompt is shown in the appendix (Appendix A). Our prompt has been designed carefully with the current literature findings of GPT-3 prompts (Reynolds and McDonell, 2021) in mind.

Specifically, the prompt follows the general template shown in the appendix, but has task-specific information to allow the large-scale language models to generalize better about the data distribution. 

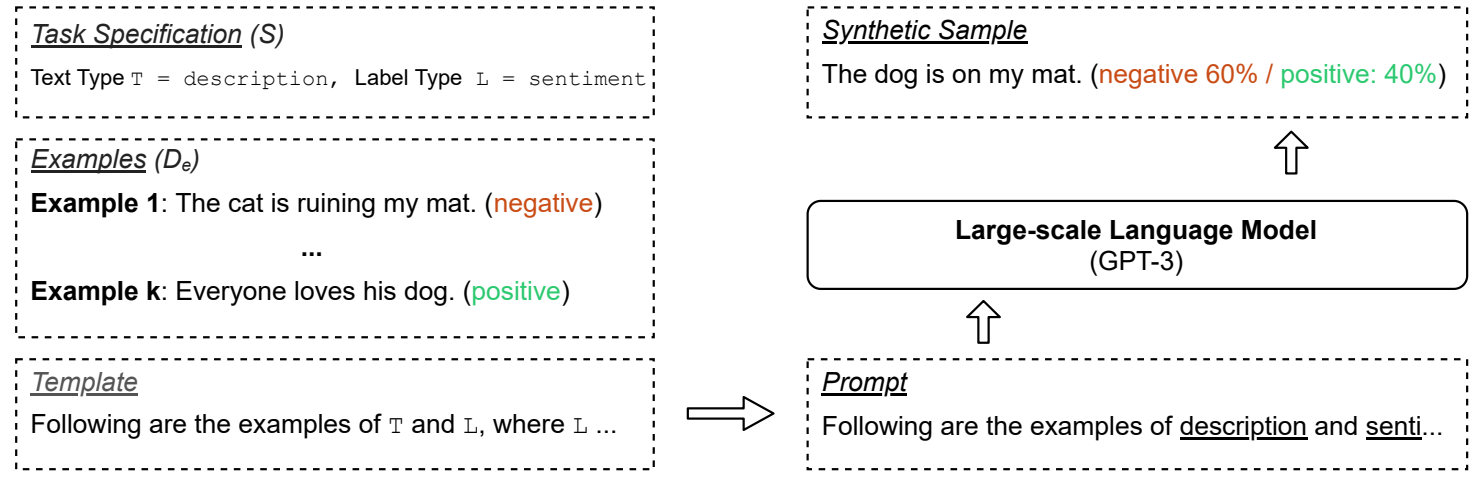

Figure 2: An illustration of GPT3Mix. The soft-labels of augmentation are extracted from the normalized labeltoken distributions predicted by the language model. Note that $v$ has been omitted in the task specification $S$ due to space limits.

These task indicators are unique to each task and provide meta-information of the task.

1. Text Type T: Meta-type of the input text $\mathrm{x}$. For example, in movie review sentiment analysis, the text type corresponds to movie review.

2. Label Type $L$ : Meta type of the label class $y$. For the example above, the label type corresponds to sentiment.

3. Label-token Verbalizer $v: \mathcal{Y} \rightarrow \mathcal{V}$ : Similar to the concept of verbalizers in the work of Schick and Schütze (2020b), the one-to-one mapping between the label classes $y \in \mathcal{Y}$ and word tokens in the language model's vocabulary $\mathcal{V}^{3}$ is needed to formulate the prompt.

The triple of the meta information above forms the task specification $S=(T, L, v)$. Each task $\mathcal{T}$ requires a task specification $S_{\mathcal{T}}$ to be able to formulate a prompt for GPT3Mix. By default, the generic task specification $S_{\text {generic }}=($ text, label, $I)$ is used to construct prompts, where $I$ is the identity function assuming that the class label exists in the vocabulary $\mathcal{V}$.

Augmentation Extraction The augmentation text $\mathbf{x}^{\prime}$ and the label $y^{\prime}$ are generated in succession after the prompt as a natural text. A predefined prompt template in the examples signals the language model to generate $\left(\mathbf{x}^{\prime}, y^{\prime}\right)$ with a structure, allowing us to extract respective values through pattern matching. Joint text and label generation

\footnotetext{
${ }^{3}$ In our implementation, we do not consider cases where a label class corresponds to multiple tokens. Regardless, expanding our work to incorporate multiple label tokens should be trivial.
}

also constraints the generated text to be associated with the correct label.

As illustrated in the prompt exhibit (Appendix A), our particular prompt design ensures that the label token that corresponds to $v\left(y^{\prime}\right)$ is generated after $\mathbf{x}$. This approach is inspired by the findings that, when inducing language models to come to a verdict, they require sufficient token lengths of "silent reasoning" prior to coming to a conclusion.

As large-scale language models are known to be few-shot learners (Brown et al., 2020), we also leverage GPT-3 to perform pseudo-labeling. The likelihood of generating the label-tokens is normalized to obtain the soft-label probability of the augmentation text $\mathbf{x}^{\prime}$. Concretely, the pseudo-label probability of an augmentation text $\mathrm{x}^{\prime}$ being labelled with label $y^{\prime}$ is as follows:

$$
p\left(y^{\prime} \mid \mathbf{x}^{\prime}\right) \propto p_{\mathrm{LM}}\left(v_{\mathcal{T}}\left(y^{\prime}\right) \mid \mathcal{P}\left(\mathbf{x}^{\prime}, S_{\mathcal{T}}\right)\right),
$$

where $p_{\mathrm{LM}}$ is the language modeling likelihood and $\mathcal{P}: \mathcal{S} \rightarrow \mathcal{X}$ is the function that constructs the prompt given a task specification.

Our approach effectively combines text perturbation, pseudo-labeling, and knowledge distillation in a single augmentation operation. In practice, augmentation samples with pseudo-labels are trained along with the real samples using the cross-entropy loss. This is in contrast to prior work, in which pseudo-labels are usually used for consistency regularization in the context of semi-supervised learning (Berthelot et al., 2019).

\section{Experiments}

We evaluate our augmentation approach on the following seven classification benchmarks: 


\begin{tabular}{|c|c|c|c|c|c|c|c|c|c|c|}
\hline \multirow[b]{2}{*}{ Dataset } & \multirow[b]{2}{*}{ Sub. } & \multicolumn{4}{|c|}{ DistilBERT $_{\text {base }}$} & \multicolumn{5}{|c|}{ BERT $_{\text {base }}$} \\
\hline & & - & EDA & BT & Ours & - & EDA & BT & TMix & Ours \\
\hline SST-2 & $\begin{array}{l}0.1 \% \\
0.3 \% \\
1.0 \%\end{array}$ & $\begin{array}{l}56.6_{4.6} \\
62.8_{6.2} \\
79.2_{3.5}\end{array}$ & $\begin{array}{l}56.7_{6.8} \\
63.1_{7.6} \\
76.9_{2.3}\end{array}$ & $\begin{array}{l}56.9_{5.6} \\
62.7_{5.8} \\
77.4_{3.8}\end{array}$ & 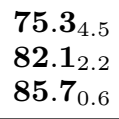 & $\begin{array}{l}57.1_{4.6} \\
65.6_{5.9} \\
82.0_{2.8}\end{array}$ & $\begin{array}{l}56.6_{4.3} \\
66.7_{5.2} \\
79.6_{1.9} \\
\end{array}$ & $\begin{array}{l}55.6_{3.8} \\
66.5_{6.4} \\
80.7_{3.1}\end{array}$ & $\begin{array}{l}56.9_{5.5} \\
64.1_{7.6} \\
79.9_{2.9}\end{array}$ & $\begin{array}{l}\mathbf{7 8 . 0}_{4.1} \\
\mathbf{8 4 . 9 _ { 1 . 4 }} \\
\mathbf{8 7 . 7 _ { 0 . 6 }}\end{array}$ \\
\hline COLA & $\begin{array}{l}0.1 \% \\
0.3 \% \\
1.0 \% \\
\end{array}$ & $\begin{array}{l}62.9_{6.3} \\
64.1_{5.7} \\
67.1_{2.3}\end{array}$ & $\begin{array}{l}57.3_{8.4} \\
58.2_{4.4} \\
59.8_{6.3}\end{array}$ & $\begin{array}{l}55.6_{6.0} \\
54.7_{7.5} \\
55.5_{5.9}\end{array}$ & $\begin{array}{l}\mathbf{6 8 . 6}_{0.1} \\
\mathbf{6 8 . 5}_{0.3} \\
\mathbf{6 8 . 6}_{0.3}\end{array}$ & $\begin{array}{l}60.7_{7.9} \\
65.5_{5.0} \\
\mathbf{7 0 . 9}_{2.3}\end{array}$ & $\begin{array}{l}60.1_{6.8} \\
63.0_{4.3} \\
63.2_{4.7} \\
\end{array}$ & $\begin{array}{l}55.2_{8.3} \\
54.2_{6.5} \\
56.6_{6.4}\end{array}$ & $\begin{array}{l}61.5_{8.9} \\
67.9_{2.3} \\
70.2_{2.0}\end{array}$ & $\begin{array}{l}\mathbf{6 8 . 6}_{0.2} \\
\mathbf{6 8 . 7} \mathbf{7}_{0.6} \\
68.5_{0.3} \\
\end{array}$ \\
\hline TREC6 & $\begin{array}{l}0.1 \% \\
0.3 \% \\
1.0 \%\end{array}$ & $\begin{array}{l}30.0_{7.2} \\
39.3_{9.2} \\
66.9_{5.8}\end{array}$ & $\begin{array}{l}30.4_{9.0} \\
37.8_{8.0} \\
62.6_{8.6}\end{array}$ & $\begin{array}{c}27.3_{6.7} \\
40.4_{10.8} \\
\mathbf{6 9 . 4}_{7.8}\end{array}$ & $\begin{array}{l}\mathbf{4 1 . 3}_{5.3} \\
\mathbf{4 7 . 9}_{4.1} \\
57.4_{2.8}\end{array}$ & $\begin{array}{l}32.1_{6.4} \\
40.7_{9.2} \\
67.0_{7.5}\end{array}$ & $\begin{array}{l}29.3_{7.1} \\
42.0_{8.1} \\
65.9_{7.1}\end{array}$ & $\begin{array}{c}30.3_{7.7} \\
39.1_{11.5} \\
69.3_{6.3}\end{array}$ & $\begin{array}{l}31.9_{8.2} \\
39.3_{6.5} \\
\mathbf{6 9 . 4}_{7.8}\end{array}$ & 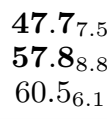 \\
\hline CR & $\begin{array}{l}0.1 \% \\
0.3 \% \\
1.0 \%\end{array}$ & $\begin{array}{l}58.0_{4.7} \\
63.1_{4.8} \\
70.8_{5.7}\end{array}$ & $\begin{array}{l}58.9_{7.9} \\
64.4_{5.2} \\
71.7_{5.4}\end{array}$ & $\begin{array}{l}58.5_{7.9} \\
61.4_{5.6} \\
70.6_{4.6}\end{array}$ & $\begin{array}{l}\mathbf{6 9 . 2}_{6.3} \\
\mathbf{7 8 . 9 _ { 3 . 2 }} \\
\mathbf{8 3 . 2 _ { 1 . 2 }}\end{array}$ & $\begin{array}{l}59.0_{4.5} \\
63.5_{6.6} \\
75.8_{4.0}\end{array}$ & $\begin{array}{l}57.9_{7.1} \\
65.3_{4.5} \\
73.9_{3.5}\end{array}$ & $\begin{array}{l}57.9_{4.5} \\
64.2_{5.5} \\
74.6_{3.7}\end{array}$ & $\begin{array}{l}58.9_{5.6} \\
63.0_{4.7} \\
72.5_{4.6}\end{array}$ & $\begin{array}{l}\mathbf{7 0 . 0}_{5.8} \\
\mathbf{8 0 . 8}_{2.4} \\
\mathbf{8 4 . 7 _ { 1 . 9 }}\end{array}$ \\
\hline SUBJ & $\begin{array}{l}0.1 \% \\
0.3 \% \\
1.0 \%\end{array}$ & $\begin{array}{l}\mathbf{8 3 . 9 _ { 2 . 5 }} \\
88.4_{1.0} \\
\mathbf{9 0 . 7 _ { 0 . 9 }}\end{array}$ & $\begin{array}{l}83.8_{3.5} \\
\mathbf{8 8 . 4} 1.3 \\
90.5_{0.9}\end{array}$ & $\begin{array}{l}81.4_{5.2} \\
87.2_{1.3} \\
90.1_{0.7}\end{array}$ & $\begin{array}{l}82.3_{6.0} \\
87.5_{1.5} \\
89.3_{1.5}\end{array}$ & $\begin{array}{l}84.1_{4.0} \\
89.3_{1.4} \\
\mathbf{9 1 . 8} \mathbf{8}_{0.8}\end{array}$ & $\begin{array}{l}84.7_{3.1} \\
89.4_{3.5} \\
91.4_{1.1}\end{array}$ & $\begin{array}{l}81.4_{7.2} \\
88.4_{1.9} \\
90.9_{0.9}\end{array}$ & $\begin{array}{l}83.6_{4.4} \\
\mathbf{8 9 . 7}_{1.3} \\
91.7_{0.9}\end{array}$ & $\begin{array}{l}\mathbf{8 5 . 4} 4.3 \\
87.5_{2.3} \\
90.6_{1.1}\end{array}$ \\
\hline MPQA & $\begin{array}{l}0.1 \% \\
0.3 \% \\
1.0 \% \\
\end{array}$ & $\begin{array}{l}66.5_{6.0} \\
77.1_{5.4} \\
84.0_{2.3}\end{array}$ & $\begin{array}{l}69.2_{5.0} \\
78.2_{4.8} \\
82.3_{2.9}\end{array}$ & $\begin{array}{l}62.3_{9.1} \\
72.9_{6.8} \\
82.2_{1.9}\end{array}$ & 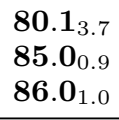 & $\begin{array}{l}65.0_{4.7} \\
71.3_{5.6} \\
83.0_{3.4}\end{array}$ & $\begin{array}{l}69.1_{4.8} \\
75.8_{3.5} \\
81.9_{1.9} \\
\end{array}$ & $\begin{array}{l}61.0_{6.8} \\
72.6_{5.9} \\
83.0_{2.4}\end{array}$ & $\begin{array}{l}65.2_{5.2} \\
74.2_{3.4} \\
83.0_{2.4}\end{array}$ & $\begin{array}{l}\mathbf{7 7 . 9}_{5.0} \\
\mathbf{8 4 . 7 _ { 1 . 0 }} \\
\mathbf{8 6 . 8 _ { 1 . 1 }} \\
\end{array}$ \\
\hline RT20 & $\begin{array}{l}0.1 \% \\
0.3 \% \\
1.0 \% \\
\end{array}$ & $\begin{array}{l}51.9_{2.6} \\
51.9_{2.5} \\
56.2_{5.7} \\
\end{array}$ & $\begin{array}{l}52.1_{2.8} \\
51.6_{3.0} \\
55.0_{3.6} \\
\end{array}$ & $\begin{array}{l}51.5_{2.6} \\
51.2_{2.2} \\
55.9_{4.1} \\
\end{array}$ & $\begin{array}{l}\mathbf{5 5 . 0}_{5.3} \\
\mathbf{6 0 . 7 _ { 4 . 5 }} \\
\mathbf{7 2 . 3}_{1.9} \\
\end{array}$ & $\begin{array}{l}50.9_{2.1} \\
51.4_{2.7} \\
57.9_{4.5} \\
\end{array}$ & $\begin{array}{l}51.8_{2.7} \\
51.9_{2.8} \\
57.9_{5.3} \\
\end{array}$ & $\begin{array}{l}53.1_{2.6} \\
51.4_{3.6} \\
57.4_{4.2} \\
\end{array}$ & $\begin{array}{l}53.2_{3.7} \\
52.0_{2.6} \\
56.0_{4.4}\end{array}$ & 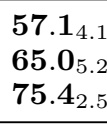 \\
\hline Average & $\begin{array}{l}0.1 \% \\
0.3 \% \\
1.0 \%\end{array}$ & $\begin{array}{l}58.5 \\
63.8 \\
73.6\end{array}$ & $\begin{array}{l}58.3 \\
63.1 \\
71.2\end{array}$ & $\begin{array}{l}56.2 \\
61.5 \\
71.6\end{array}$ & $\begin{array}{l}67.4 \\
72.9 \\
77.5\end{array}$ & $\begin{array}{l}58.4 \\
63.9 \\
75.5\end{array}$ & $\begin{array}{l}58.5 \\
64.9 \\
73.4\end{array}$ & $\begin{array}{l}56.4 \\
62.4 \\
73.2\end{array}$ & $\begin{array}{l}58.7 \\
64.3 \\
74.7\end{array}$ & $\begin{array}{l}69.2 \\
75.6 \\
79.2\end{array}$ \\
\hline
\end{tabular}

Table 1: Main data augmentation results on $0.1 \%, 0.3 \%$, and $1.0 \%$ training set sub-sample levels. We compare different augmentation strategies by transformer architectures on the downstream classification performance. Experiments have been repeated 10 times and the statistics are presented in the mean std $_{\text {format. }}$

SST-2 (Socher et al., 2013) is a sentiment classification dataset that contains movie reviews crawled from Rotten Tomatoes and their corresponding binary labels. CR (Hu and Liu, 2004) dataset is a set of Amazon product reviews labeled by binary sentiments. The Corpus of Linguistic Acceptability (COLA) (Warstadt et al., 2018) is a collection of sentences extracted from publications annotated with grammaticality. The TREC6 dataset (Voorhees and Tice, 1999) concerns the question classification task consisting of open-domain, factbased questions divided into broad semantic categories. MPQA (Wiebe et al., 2005) consists of opinions and their semantic polarity. The subjectivity dataset (SUBJ) (Pang and Lee, 2004) contains movie reviews labeled with objectivity.

RT20 is the newly proposed benchmark with which we perform controlled experiments on language models. The dataset is a binary sentiment classification corpus, collected from Rotten Tomatoes accessed after a certain date. The details about the collection and preparation is provided in Appendix C.

\subsection{Experimental Settings}

To showcase our approach, we conduct downstream classification experiments on artificially data-scarce tasks by sub-sampling the training set. For each experiment, we perform a class-balanced sub-sample on the training set. We account for statistical variance in our experiments by fixating the sub-samples on 15 different data seeds and repeating the augmentation procedure and downstream classification experiments on all sub-samples. The data seeds were chosen randomly ${ }^{4}$.

For the classifier architecture, we use the base size BERT (Devlin et al., 2019) and DistilBERT (Sanh et al., 2019) models, which have 109M and $67 \mathrm{M}$ parameters respectively. For each downstream classification trial, we initialize the classifier model with the pre-trained parameters provided by the Huggingface Transformers library (Wolf et al., 2019) and randomly initialize the classifier layers, which consist of two fully connected layers that predict the class labels from the output embeddings

\footnotetext{
${ }^{4}$ The data seeds were randomly generated using a master seed.
} 


\begin{tabular}{c|cccc}
\hline & \multicolumn{4}{|c}{ BERT $_{\text {large }}$} \\
\hline Sub. & - & EDA & BT & Ours \\
\hline $0.1 \%$ & $60.3_{7.9}$ & $63.8_{7.2}$ & $63.4_{7.4}$ & $\mathbf{8 4 . 0}_{4.4}$ \\
$0.3 \%$ & $74.1_{8.9}$ & $73.2_{5.8}$ & $73.1_{9.4}$ & $\mathbf{8 8 . 7}_{1.0}$ \\
$1.0 \%$ & $87.8_{1.5}$ & $87.3_{1.4}$ & $87.0_{2.7}$ & $\mathbf{9 0 . 8 _ { 0 . 6 }}$ \\
\hline
\end{tabular}

Table 2: Additional data augmentation experiments on SST-2 with BERT $_{\text {large, }}$ which has $335 \mathrm{M}$ parameters. The larger network capacity enables the model to better exploit the GPT3Mix augmentations, allowing it to match the performance of BERT base $_{\text {trained on the full }}$ data with just $1.0 \%$ subsample of the training data.

of the transformer architectures. The classifiers are trained automatically by employing early stopping against the validation score with patience of 20 training epochs. We report classification accuracies in all of our tables.

\subsection{Implementation Details}

For selecting the optimal task specification for each task in GPT3Mix augmentation, we evaluated the performance of few handcrafted task specification candidates on the validation set and chose the highest performing one. The details about the optimal task specifications are presented in Appendix B. The inference on GPT-3 was carried out via the OpenAI API Beta Access program. We used the largest GPT-3 model available on (davinci) unless otherwise stated. On average, a GPT3Mix augmentation roughly consumes 300 tokens in combined length (prompt and generation). For GPT-3 generation, top-p and the temperature was set to 1 and the frequency penalty was set to 0.02 (Holtzman et al., 2019). The augmentation ratio between the training set and the synthetic set was set to 10 unless otherwise stated.

During classifier training, we used the Adam optimizer with decoupled weight decay (Kingma and Ba, 2014; Loshchilov and Hutter, 2017) and a learning rate of $3 e-5$. The learning rate had a warm-up period of 3 epochs. PyTorch and M40 GPUs were used to run the experiments.

\subsection{Data Augmentation Experiments}

We compare our approach to Easy Data Augmentation (EDA) (Wei and Zou, 2019), back-translation (BT) (Fadaee et al., 2017), and TMix (Chen et al., 2020). For the back-translation baseline, texts were translated to and from German using Transformer architectures trained on the WMT16 English-German corpus provided by Fairseq (Ott

\begin{tabular}{c|cccc}
\hline & \multicolumn{4}{|c}{$k$} \\
\hline Sub. & 1 & 2 & 4 & 8 \\
\hline $0.1 \%$ & $65.5_{3.3}$ & $71.2_{6.5}$ & $74.6_{3.9}$ & $72.0_{6.7}$ \\
$0.3 \%$ & $78.9_{3.9}$ & $80.0_{2.7}$ & $80.2_{2.1}$ & $80.0_{1.6}$ \\
$1.0 \%$ & $85.2_{0.6}$ & $84.3_{0.7}$ & $84.3_{0.7}$ & $84.2_{1.2}$ \\
\hline
\end{tabular}

Table 3: An ablation study on the number of examples $k$ in GPT3Mix prompts. When $k=1$, GPT-3 produces point-wise perturbed samples. Experiments are carried out on the SST-2 dataset.

et al., 2019). For TMix, we employ the hyperparameters reported by the authors. We compare with

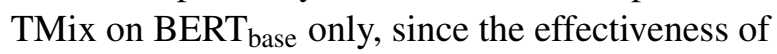
TMix is not established in other architectures ${ }^{5}$.

The results on data-scarce text augmentation are presented in Table 1. First, we notice that, in most cases, our approach outperforms other augmentation baselines by a large margin. Also, our approach achieves higher stability in terms of the variance of repeated trials and inter-task fluctuations than other augmentation methods. Although back-translation and EDA do outperform GPT3Mix in certain configurations, GPT3Mix offers the most consistent performance boost for the downstream classifiers across all tasks. This is evident from the average classification accuracies of all tasks, in which GPT3Mix improves the baseline as much as $18.6 \%$ (for $\mathrm{BERT}_{\text {base }}$ ) while other methods show nearly no improvement ${ }^{6}$.

We also note that, despite non-augmented baselines of DistilBERT ${ }_{\text {base }}$ and BERT base $_{\text {being very }}$ close (58.5 and 58.4 respectively on average of $0.10 \%$ subsamples), a much larger augmentation effect is observed in BERT base results $(67.4 \rightarrow 69.2)$. Improving model robustness is known to require significantly larger model complexity (Ye et al., 2019), hence $\mathrm{BERT}_{\text {base }}$, having $65 \%$ more parameters than DistilBERT base $_{\text {, }}$ utilizes GPT3Mix samples better than the counterpart. This effect is more apparent in the even larger model (Table 2), which outperforms fully trained BERT $\mathrm{Base}_{\text {bith }}$ wust $1 \%$ of the original data.

Furthermore, we observe that augmenting with GPT3Mix significantly improves the baseline across all subsamples of RT20, eliminating the suspicion that the data augmentation effect of GPT3Mix is attributed to data memorization of

\footnotetext{
${ }^{5}$ Our attempt on searching TMix hyperparameters for

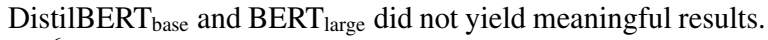

${ }^{6} \mathrm{We}$ employed the hyperparameters proposed by the authors of EDA and BT.
} 


\begin{tabular}{c|cccc}
\hline & \multicolumn{4}{|c}{ Model Size } \\
\hline Sub. & ada & babbage & curie & davinci \\
\hline $0.1 \%$ & $61.9_{4.1}$ & $65.2_{6.9}$ & $65.9_{5.3}$ & $67.6_{7.2}$ \\
$0.3 \%$ & $74.6_{4.8}$ & $69.7_{7.3}$ & $74.6_{4.5}$ & $78.3_{2.9}$ \\
$1.0 \%$ & $81.6_{1.0}$ & $82.5_{1.1}$ & $83.4_{1.8}$ & $84.3_{1.1}$ \\
\hline
\end{tabular}

Table 4: An ablation study on the size of the language model with the SST-2 dataset. Larger language models provide greater augmentation benefits in data-limited environment.

GPT-3. Also note that, due to the recency of the RT20 dataset, the pretrained classification transformers do not perform as well as on the older counterpart, SST-2. However, GPT3Mix is able to alleviate the difficulty through knowledge distillation and mix-based robust training.

Full Dataset Experiments We also perform full dataset data augmentation experiments to confirm that GPT3Mix still offers benefits even when taskspecific data are abundant. We augmented the full SST-2 dataset with one-to-one ratio of synthetic samples from GPT3Mix, and the experiments show that GPT3Mix improves the accuracy of DistilBERT base $_{\text {from }} 90.28 \%$ to $90.70 \%(0.42 \%$ improvement) and the accuracy of BERT $\mathrm{Base}_{\text {bas }}$ from $90.33 \%$ to $93.25 \%$ (2.92\% improvement). Again, we observe a larger improvement in the more expressive model, in align with previous findings (Zhang et al., 2017; Shafahi et al., 2019).

\subsection{Ablation Studies}

We conduct a number of ablation experiments to study the underlying mechanism of GPT3Mix. Note that the augmentation results for GPT3mix in the following ablation studies may underperform compared to the results presented in $\$ 4.3$ due to ablation studies having lower augmentation ratios and using smaller language models (curie). Also note that all ablation experiments were carried out on the DistilBERT base $_{\text {classifier architecture. }}$

\subsubsection{Number of Prompt Examples}

First, the effect of the number of examples in GPT3Mix prompts $(k)$ on the downstream augmentation performance is studied. GPT3Mix requires $k \geq 2$ to effectively mix existing samples and generate interpolated text samples. However, supplying one example $(k=1)$ per prompt and expecting GPT-3 to introduce perturbations or paraphrases of the given example can be a viable strategy. We vary $k$ on the SST-2 dataset and observe the downstream

\begin{tabular}{c|cccc}
\hline & \multicolumn{4}{|c}{ Pre-trained Language Model } \\
\hline Sub. & - & GPT-2 & GPT-neo & davinci \\
\hline $0.1 \%$ & $56.6_{4.6}$ & $64.1_{6.5}$ & $71.3_{4.7}$ & $\mathbf{7 5 . 3}$ \\
$0.3 \%$ & $62.8_{6.2}$ & $76.9_{3.6}$ & $80.2_{1.9}$ & $\mathbf{8 2 . 1}_{2.2}$ \\
$1.0 \%$ & $79.2_{3.5}$ & $76.1_{3.6}$ & $82.6_{1.1}$ & $\mathbf{8 5 . 7 _ { 0 . 6 }}$ \\
\hline
\end{tabular}

Table 5: Open-source alternatives are compared to the largest GPT-3 model on the SST-2 dataset. For GPT-2, the large version that has $774 \mathrm{M}$ parameters was used. For GPT-neo, the smaller version of $1.3 \mathrm{~B}$ parameters was used.

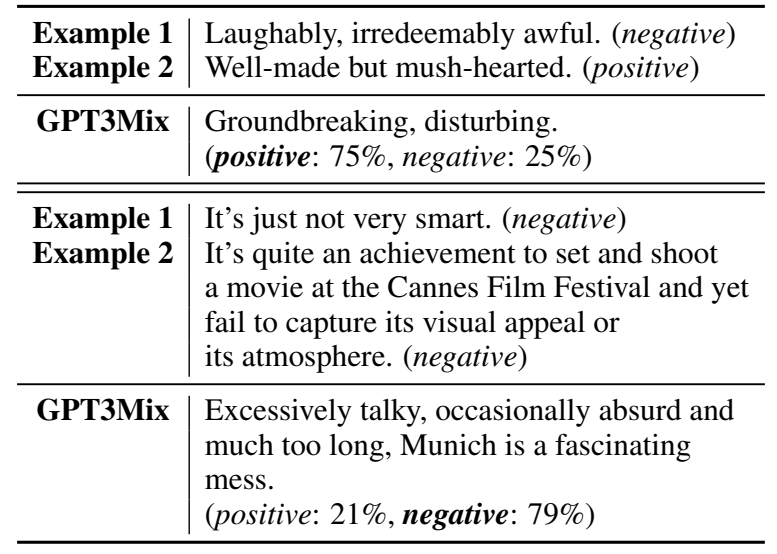

Table 6: SST-2 augmentation samples from GPT3Mix (davinci). GPT3Mix annotates synthetic samples with soft-labels predicted by the language model.

performances (Table 3). The second-largest GPT-3 model (curie) was used and the augmentation multiplier was set to 10 .

From the results, we notice that when the data availability is severely limited (i.e. $0.1 \%$ and $0.3 \%$ ), point-wise perturbation doesn't offer the performance improvement as much as when $k \geq 2$. However, as data becomes more abundant, increasing the number of mixing samples offers marginally small benefits for data augmentation. Yet, increasing the number of examples incurs additional overhead to the GPT-3 inference cost.

Generally, over-providing prompt examples may constraint the degrees of freedom and causing the synthetic samples to overfit on the data, hurting the downstream performances. However, a significant improvement from $k=2$ to $k=4$ is observed for the $0.1 \%$ sub-sample level. In our data augmentation studies, we weigh in on $k=2$ as a reasonable balance between the trade-off between GPT-3 inference costs and performance gains. 


\begin{tabular}{c|ccc}
\hline Sub. & No Aug. & Hard Labels & Soft-labels \\
\hline $0.1 \%$ & $55.8_{5.1}$ & $61.6_{8.0}$ & $71.2_{6.5}$ \\
$0.3 \%$ & $64.9_{8.0}$ & $67.7_{5.9}$ & $80.0_{2.7}$ \\
$1.0 \%$ & $77.9_{3.6}$ & $79.0_{2.8}$ & $84.3_{0.7}$ \\
\hline
\end{tabular}

Table 7: An ablation study on the employment of pseudo-labels. Hard labels are obtained from the beam search of the entire sequence autoregressively generated by the language model.

\subsubsection{Language Model Capacity}

Next, we study the influence of the model capacity of the augmenting language model on the quality of augmentations. OpenAI offers GPT- 3 in four different capacities: ada, babbage, curie, and davinci ${ }^{7}$, listed in the increasing order of model complexity. In this study, the augmentation ratio is set to 5. The results (Table 4) show that having larger and more expressive language models benefit data augmentation.

Additionally, we conduct comparative experiments to verify whether open-source alternatives to GPT-3 could still provide comparable performance gains through data augmentation. As opensource alternatives, GPT-2 (Radford et al.) and GPT-neo (Black et al., 2021) were chosen. The latter is a popular alternative to the commercial GPT-3, performing competitively with the smaller versions (ada and babbage) of the counterpart. Our results (Table 5) show that the open-source GPT-like models still provide comparable performance gains, strongly suggesting that our promptbased GPT3Mix approach can be versatile in the choice of pre-trained language models. Even the smaller GPT-2 model could provide performance gains.

\subsubsection{Task Specification}

We are also interested in how the design choice of task specification for prompt construction affects the downstream performance. To analyze the effect, we compare the optimal task specification $S_{\mathcal{T}}$ to a generic one $\left(S_{\text {generic }}\right)$, where the nature of the task cannot be inferred from the description. For this study, we used curie as the augmenting language model with an augmentation ratio of 3 . The results in Table 8 support our conjecture that the language model utilizes the meta-information about the dataset to generate better data samples,

\footnotetext{
${ }^{7}$ The sizes of the language models are known to be $2.7 \mathrm{~B}$, $6.7 \mathrm{~B}, 13 \mathrm{~B}$, and $175 \mathrm{~B}$ respectively; however, OpenAI has not officially disclosed the exact numbers yet.
}

\begin{tabular}{c|c|ccc}
\hline Dataset & Sub. & No Aug. & $S_{\text {generic }}$ & $S_{\mathcal{T}^{\star}}$ \\
\hline \multirow{3}{*}{ SST-2 } & $0.1 \%$ & $55.8_{5.1}$ & $60.1_{5.2}$ & $71.2_{6.5}$ \\
& $0.3 \%$ & $64.9_{8.0}$ & $72.6_{5.7}$ & $80.0_{2.7}$ \\
& $1.0 \%$ & $77.9_{3.6}$ & $81.4_{1.7}$ & $84.3_{0.7}$ \\
\hline \multirow{3}{*}{ COLA } & $0.1 \%$ & $64.9_{4.7}$ & $68.4_{0.4}$ & $68.6_{0.0}$ \\
& $0.3 \%$ & $62.2_{7.2}$ & $65.7_{2.7}$ & $68.7_{0.2}$ \\
& $1.0 \%$ & $67.8_{1.6}$ & $68.7_{0.3}$ & $69.1_{1.1}$ \\
\hline
\end{tabular}

Table 8: An ablation study on task specifications. $S_{\text {generic }}$ denotes a generic task specification that does not hold task-specific meta-information ( $\$ 3)$, and $S_{\mathcal{T}}$ * denotes the optimal specification for the corresponding task.

and thus prompt designs have a significant impact on the augmentation quality. However, the generic task specification outperforms other augmentation baselines, highlighting the effectiveness of employing large-scale language models as the augmentation source.

\subsubsection{Pseudo-labeling}

Finally, we study the effect of employing pseudolabels from the label token probabilities predicted by the large-scale language model. we compare the augmentation performance when the label tokens optimized from the sequence-wide beam search are used instead. Results on SST-2 (Table 7) show that employing soft-labels has a strong advantage over sequence-optimized labels. The performance gap between the hard and soft-labels can be considered as the benefit of utilizing the class distribution jointly predicted by the language model as a form of knowledge distillation for synthetic samples (Kim and Rush, 2016). curie was used as the GPT-3 model with the augmentation ratio of 5 .

\subsubsection{Qualitative Analysis}

Language models are known to be sensitive to the selection and the order of the examples presented in the prompt, causing biases in the predictions (Zhao et al., 2021; Reynolds and McDonell, 2021). Our proposed method hinges on this unique property of large-scale language models, hence we wish to qualitatively examine the augmentation samples to further support our hypothesis.

The augmentation samples for the SST-2 dataset are presented in Table 6. First, we notice that the synthetic sentiment is correlated with the input sentiments. If both examples are either all negative (the second example), the sentiment of the augmentation sample is heavily biased towards negative. Second, we also discover that the augmented 
sample follows the similar syntactic and semantic structure of the example texts. As demonstrated in the first case, the short and phrasal structure of the examples is well translated into the generated sample, supporting the notion that language models are able to learn from in-context examples even for generation and pseudo-labeling tasks. In the second example, the linguistic similarity between the generated sample and the given examples is more abstract (use of adjective phrases and enumerated clauses), suggesting that language models are capable of creative interpolation.

\section{Conclusion}

In this paper, we proposed a novel text augmentation technique that leverages large-scale language models and their abilities to perform controlled generation via prompts. Our extensive experiments on classification tasks show that our augmentation method can improve robustness of pretrained transformers through mix-based perturbation and knowledge distillation without the online inference on heavy LMs. Thus, our method can be a competitive alternative to prompt-based task-solving (Brown et al., 2020) or direct fine-tuning (Liu et al., 2021). As future work, we are interested in the possibility of further pushing the boundaries of state-of-the-art architectures via GPT3Mix. We are also working towards improving generation efficiency by optimizing example selection and prompt templates.

\section{Ethical Considerations}

Our approach presents several ethical challenges. Pre-trained language models that are trained on untreated corpora are known to exhibit social biases (Bordia and Bowman, 2019; Hutchinson et al., 2020; Abid et al., 2021; Bender et al., 2021) and toxicity (Gehman et al., 2020). The biased property is concerning because language models are prone to degeneration even in the absence of bias or toxicity in the prompts (Gehman et al., 2020). As a result, GPT3Mix is not exempt from the possibility of propagating linguistic biases and toxicity even if the real training examples were ensured to be unbiased. Furthermore, linguistic bias could be amplified through iterative applications of GPT3Mix (i.e., using GPT3Mix-augmented samples as the source examples for the next iteration of GPT3Mix).

To address these issues, we propose three remedies to reduce the concerns. First, debiased pretrained language models can be used in place of
GPT-3. Language models can be adapted to debiased and non-toxic corpora (Gehman et al., 2020) or treated with modifications to the word embedding space (Basta and Costa-jussà, 2021) to inhibit their tendency to generate bias. Moreover, GPT3Mix has been shown to work well with various pre-trained language models (Table 5). Second, specific decoding strategies can be employed to reduce bias at inference time. Recent body of work has shown that handcrafted dictionaries can be employed to suppress the selection of offensive words (Gehman et al., 2020) and that language models can implicitly learn to identify biases through self-diagnosis, which can be exploited for self-debiasing (Schick et al., 2021). Third, humanin-the-loop in the augmentation process can be utilized to manually identify and filter linguistic bias.

Note that the ethical implications can be minimized by using GPT3Mix only for augmenting discriminators, where the augmented samples are removed once the training process is complete. However, for the general purpose of populating datasets, linguistic bias is of ethical concern and can be alleviated using the existing work on debiasing.

\section{Acknowledgement}

We would like to show appreciation for the valuable feedback given by Jung-Woo Ha, Gyuwan Kim, and Hwaran Lee through detailed reviews. We also thank Jaimeen Ahn for providing comments on ethical considerations of our approach and the underlying language model.

\section{References}

Abubakar Abid, Maheen Farooqi, and James Zou. 2021. Persistent anti-muslim bias in large language models. arXiv preprint arXiv:2101.05783.

Christine Basta and Marta R Costa-jussà. 2021. Impact of gender debiased word embeddings in language modeling. arXiv preprint arXiv:2105.00908.

Emily M Bender, Timnit Gebru, Angelina McMillanMajor, and Shmargaret Shmitchell. 2021. On the dangers of stochastic parrots: Can language models be too big? In Proceedings of the 2021 ACM Conference on Fairness, Accountability, and Transparency, pages 610-623.

David Berthelot, Nicholas Carlini, Ian Goodfellow, Nicolas Papernot, Avital Oliver, and Colin Raffel. 2019. Mixmatch: A holistic approach to semi-supervised learning. arXiv preprint arXiv:1905.02249. 
Sid Black, Leo Gao, Phil Wang, Connor Leahy, and Stella Biderman. 2021. GPT-Neo: Large scale autoregressive language modeling with meshtensorflow.

Shikha Bordia and Samuel Bowman. 2019. Identifying and reducing gender bias in word-level language models. In Proceedings of the 2019 Conference of the North American Chapter of the Association for Computational Linguistics: Student Research Workshop, pages 7-15.

Samuel Bowman, Luke Vilnis, Oriol Vinyals, Andrew Dai, Rafal Jozefowicz, and Samy Bengio. 2016. Generating sentences from a continuous space. In Proceedings of The 20th SIGNLL Conference on Computational Natural Language Learning, pages 10-21.

Tom B Brown, Benjamin Mann, Nick Ryder, Melanie Subbiah, Jared Kaplan, Prafulla Dhariwal, Arvind Neelakantan, Pranav Shyam, Girish Sastry, Amanda Askell, et al. 2020. Language models are few-shot learners. arXiv preprint arXiv:2005.14165.

Jiaao Chen, Zichao Yang, and Diyi Yang. 2020. Mixtext: Linguistically-informed interpolation of hidden space for semi-supervised text classification. In Proceedings of the 58th Annual Meeting of the Association for Computational Linguistics, pages 21472157.

Jacob Devlin, Ming-Wei Chang, Kenton Lee, and Kristina Toutanova. 2019. Bert: Pre-training of deep bidirectional transformers for language understanding. In Proceedings of the 2019 Conference of the North American Chapter of the Association for Computational Linguistics: Human Language Technologies, Volume 1 (Long and Short Papers), pages 4171-4186.

Marzieh Fadaee, Arianna Bisazza, and Christof Monz. 2017. Data augmentation for low-resource neural machine translation. In Proceedings of the 55th Annual Meeting of the Association for Computational Linguistics (Volume 2: Short Papers), pages 567573.

Samuel Gehman, Suchin Gururangan, Maarten Sap, Yejin Choi, and Noah A Smith. 2020. Realtoxicityprompts: Evaluating neural toxic degeneration in language models. arXiv preprint arXiv:2009.11462.

Demi Guo, Yoon Kim, and Alexander M Rush. 2020 Sequence-level mixed sample data augmentation. In Proceedings of the 2020 Conference on Empirical Methods in Natural Language Processing (EMNLP), pages 5547-5552.

Geoffrey Hinton, Oriol Vinyals, and Jeff Dean. 2015. Distilling the knowledge in a neural network. stat, 1050:9.

Ari Holtzman, Jan Buys, Li Du, Maxwell Forbes, and Yejin Choi. 2019. The curious case of neural text degeneration. arXiv preprint arXiv:1904.09751.
Yutai Hou, Yijia Liu, Wanxiang Che, and Ting Liu. 2018. Sequence-to-sequence data augmentation for dialogue language understanding. In Proceedings of the 27th International Conference on Computational Linguistics, pages 1234-1245.

Minqing $\mathrm{Hu}$ and Bing Liu. 2004. Mining and summarizing customer reviews. In Proceedings of the tenth ACM SIGKDD international conference on Knowledge discovery and data mining, pages 168-177.

Ben Hutchinson, Vinodkumar Prabhakaran, Emily Denton, Kellie Webster, Yu Zhong, and Stephen Denuyl. 2020. Social biases in nlp models as barriers for persons with disabilities. arXiv preprint arXiv:2005.00813.

Zhengbao Jiang, Frank F Xu, Jun Araki, and Graham Neubig. 2020. How can we know what language models know? Transactions of the Association for Computational Linguistics, 8:423-438.

Yoon Kim and Alexander M Rush. 2016. Sequencelevel knowledge distillation. In Proceedings of the 2016 Conference on Empirical Methods in Natural Language Processing, pages 1317-1327.

Diederik P Kingma and Jimmy Ba. 2014. Adam: A method for stochastic optimization. arXiv preprint arXiv:1412.6980.

Varun Kumar, Ashutosh Choudhary, and Eunah Cho. 2020. Data augmentation using pre-trained transformer models. In Proceedings of the 2nd Workshop on Life-long Learning for Spoken Language Systems, pages 18-26.

Dong-Hyun Lee et al. 2013. Pseudo-label: The simple and efficient semi-supervised learning method for deep neural networks. In Workshop on challenges in representation learning, ICML, volume 3.

Mike Lewis, Yinhan Liu, Naman Goyal, Marjan Ghazvininejad, Abdelrahman Mohamed, Omer Levy, Veselin Stoyanov, and Luke Zettlemoyer. 2020. Bart: Denoising sequence-to-sequence pretraining for natural language generation, translation, and comprehension. In Proceedings of the 58th Annual Meeting of the Association for Computational Linguistics, pages 7871-7880.

Yitong Li, Trevor Cohn, and Timothy Baldwin. 2017. Robust training under linguistic adversity. In Proceedings of the 15th Conference of the European Chapter of the Association for Computational Linguistics: Volume 2, Short Papers, pages 21-27.

Xiao Liu, Yanan Zheng, Zhengxiao Du, Ming Ding, Yujie Qian, Zhilin Yang, and Jie Tang. 2021. Gpt understands, too. arXiv preprint arXiv:2103.10385.

Ilya Loshchilov and Frank Hutter. 2017. Decoupled weight decay regularization. arXiv preprint arXiv:1711.05101. 
Nathan Ng, Kyunghyun Cho, and Marzyeh Ghassemi. 2020. Ssmba: Self-supervised manifold based data augmentation for improving out-of-domain robustness. arXiv preprint arXiv:2009.10195.

Myle Ott, Sergey Edunov, Alexei Baevski, Angela Fan, Sam Gross, Nathan Ng, David Grangier, and Michael Auli. 2019. fairseq: A fast, extensible toolkit for sequence modeling. In Proceedings of NAACL-HLT 2019: Demonstrations.

Bo Pang and Lillian Lee. 2004. A sentimental education: Sentiment analysis using subjectivity summarization based on minimum cuts. In Proceedings of the 42nd Annual Meeting of the Association for Computational Linguistics (ACL-04), pages 271-278.

Mary Phuong and Christoph Lampert. 2019. Towards understanding knowledge distillation. In International Conference on Machine Learning, pages 5142-5151. PMLR.

Alec Radford, Jeffrey Wu, Rewon Child, David Luan, Dario Amodei, Ilya Sutskever, et al. Language models are unsupervised multitask learners.

Scott Reed, Honglak Lee, Dragomir Anguelov, Christian Szegedy, Dumitru Erhan, and Andrew Rabinovich. 2014. Training deep neural networks on noisy labels with bootstrapping. arXiv preprint arXiv:1412.6596.

Laria Reynolds and Kyle McDonell. 2021. Prompt programming for large language models: Beyond the few-shot paradigm. arXiv preprint arXiv:2102.07350.

Victor Sanh, Lysandre Debut, Julien Chaumond, and Thomas Wolf. 2019. Distilbert, a distilled version of bert: smaller, faster, cheaper and lighter. arXiv preprint arXiv:1910.01108.

Timo Schick and Hinrich Schütze. 2020a. Exploiting cloze questions for few-shot text classification and natural language inference. arXiv preprint arXiv:2001.07676.

Timo Schick and Hinrich Schütze. 2020b. It's not just size that matters: Small language models are also few-shot learners. arXiv preprint arXiv:2009.07118.

Timo Schick, Sahana Udupa, and Hinrich Schütze. 2021. Self-diagnosis and self-debiasing: A proposal for reducing corpus-based bias in nlp. arXiv preprint arXiv:2103.00453.

Ali Shafahi, Mahyar Najibi, Amin Ghiasi, Zheng $\mathrm{Xu}$, John Dickerson, Christoph Studer, Larry S Davis, Gavin Taylor, and Tom Goldstein. 2019. Adversarial training for free! arXiv preprint arXiv:1904.12843.

Taylor Shin, Yasaman Razeghi, Robert L Logan IV, Eric Wallace, and Sameer Singh. 2020. Autoprompt: Eliciting knowledge from language models with automatically generated prompts. arXiv preprint arXiv:2010.15980.

Mohammad Shoeybi, Mostofa Patwary, Raul Puri, Patrick LeGresley, Jared Casper, and Bryan Catanzaro. 2019. Megatron-lm: Training multi-billion parameter language models using model parallelism. arXiv preprint arXiv:1909.08053.

Richard Socher, Alex Perelygin, Jean Wu, Jason Chuang, Christopher D Manning, Andrew Y Ng, and Christopher Potts. 2013. Recursive deep models for semantic compositionality over a sentiment treebank. In Proceedings of the 2013 conference on empirical methods in natural language processing, pages 1631-1642.

Lichao Sun, Congying Xia, Wenpeng Yin, Tingting Liang, S Yu Philip, and Lifang He. 2020. Mixuptransformer: Dynamic data augmentation for nlp tasks. In Proceedings of the 28th International Conference on Computational Linguistics, pages 34363440 .

Raphael Tang, Yao Lu, Linqing Liu, Lili Mou, Olga Vechtomova, and Jimmy Lin. 2019. Distilling taskspecific knowledge from bert into simple neural networks. arXiv preprint arXiv:1903.12136.

Ellen M Voorhees and Dawn M Tice. 1999. The trec-8 question answering track evaluation. In TREC, volume 1999, page 82. Citeseer.

Alex Warstadt, Amanpreet Singh, and Samuel R Bowman. 2018. Neural network acceptability judgments. arXiv preprint arXiv:1805.12471.

Jason Wei and Kai Zou. 2019. Eda: Easy data augmentation techniques for boosting performance on text classification tasks. In Proceedings of the 2019 Conference on Empirical Methods in Natural Language Processing and the 9th International Joint Conference on Natural Language Processing (EMNLPIJCNLP), pages 6383-6389.

Janyce Wiebe, Theresa Wilson, and Claire Cardie. 2005. Annotating expressions of opinions and emotions in language. Language resources and evaluation, 39(2):165-210.

Thomas Wolf, Lysandre Debut, Victor Sanh, Julien Chaumond, Clement Delangue, Anthony Moi, Pierric Cistac, Tim Rault, Rémi Louf, Morgan Funtowicz, et al. 2019. Huggingface's transformers: State-of-the-art natural language processing. arXiv preprint arXiv: 1910.03771 .

Congying Xia, Caiming Xiong, Philip Yu, and Richard Socher. 2020a. Composed variational natural language generation for few-shot intents. arXiv preprint arXiv:2009.10056.

Congying Xia, Chenwei Zhang, Hoang Nguyen, Jiawei Zhang, and Philip Yu. 2020b. Cg-bert: Conditional text generation with bert for generalized few-shot intent detection. arXiv preprint arXiv:2004.01881. 
Qizhe Xie, Zihang Dai, Eduard Hovy, Thang Luong, and Quoc Le. 2020. Unsupervised data augmentation for consistency training. Advances in Neural Information Processing Systems, 33.

Shaokai Ye, Kaidi Xu, Sijia Liu, Hao Cheng, JanHenrik Lambrechts, Huan Zhang, Aojun Zhou, Kaisheng Ma, Yanzhi Wang, and Xue Lin. 2019. Adversarial robustness vs. model compression, or both? In Proceedings of the IEEE/CVF International Conference on Computer Vision, pages 111120.

Kang Min Yoo, Youhyun Shin, and Sang-goo Lee. 2019. Data augmentation for spoken language understanding via joint variational generation. In Proceedings of the AAAI conference on artificial intelligence, volume 33, pages 7402-7409.

Hongyi Zhang, Moustapha Cisse, Yann N Dauphin, and David Lopez-Paz. 2017. mixup: Beyond empirical risk minimization. arXiv preprint arXiv:1710.09412.

Xiang Zhang, Junbo Zhao, and Yann LeCun. 2016 Character-level convolutional networks for text classification.

Tony Z Zhao, Eric Wallace, Shi Feng, Dan Klein, and Sameer Singh. 2021. Calibrate before use: Improving few-shot performance of language models. arXiv preprint arXiv:2102.09690. 


\section{A Prompts}

The GPT3Mix prompt uses the following template. The template corresponds to the prompt-constructing function $\mathcal{P}$, which require a task specification $S_{\mathcal{T}}=(T, L, v)$.

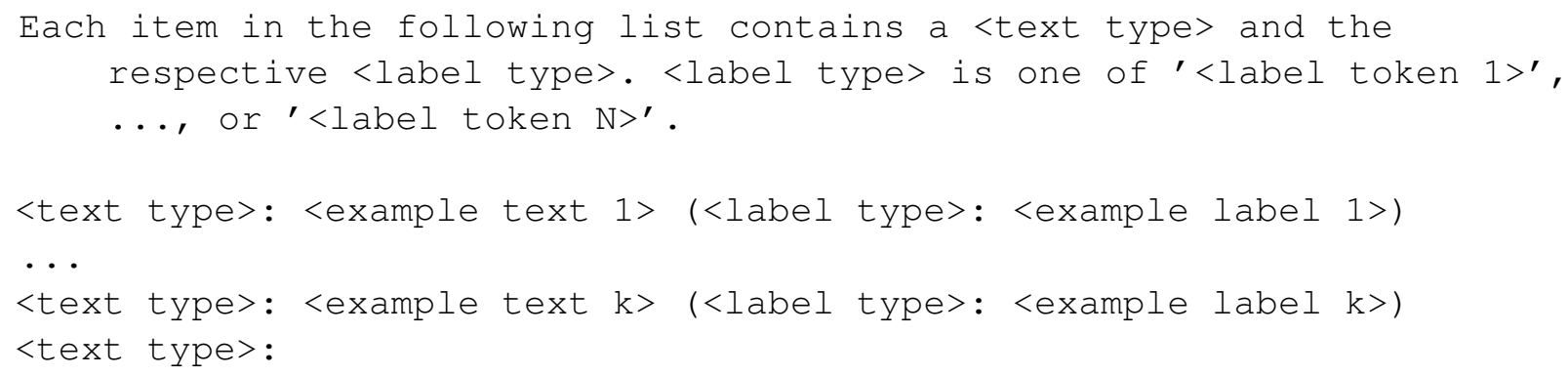

For example, given $S_{\mathrm{SST} 2}=$ (movie review, sentiment, $I$ ), the constructed GPT3Mix prompt is as follows.

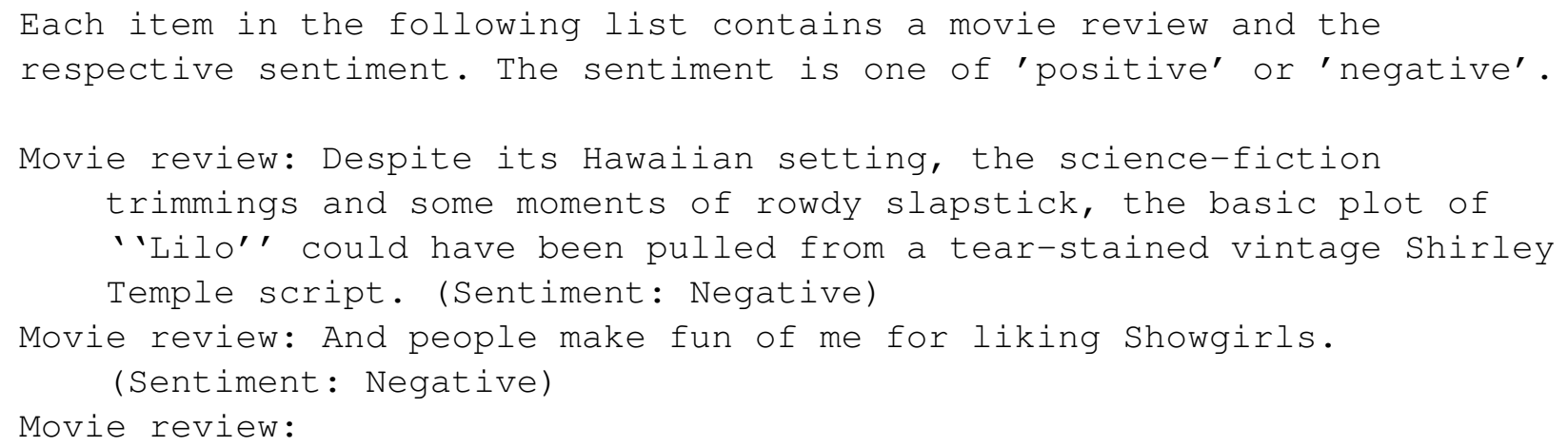

\section{B Task Specifications}

\begin{tabular}{c|ccc}
\hline Dataset & $T$ & $L$ & $v$ \\
\hline Generic & text & label & $\cdot \rightarrow \cdot$ \\
SST-2 & movie review & sentiment & pos $\rightarrow$ positive, neg $\rightarrow$ negative \\
CR & customer review & sentiment & pos $\rightarrow$ positive, neg $\rightarrow$ negative \\
SUBJ & text & objective & subjective $\rightarrow$ no, objective $\rightarrow$ yes \\
COLA & text & grammar & acceptable $\rightarrow$ correct, unacceptable $\rightarrow$ incorrect \\
TREC6 & question & type & ABBR $\rightarrow$ abbreviation, LOC $\rightarrow$ location, \\
& & & DESC $\rightarrow$ description, NUM $\rightarrow$ numeric \\
& & & ENTY $\rightarrow$ entity, HUM $\rightarrow$ human \\
MPQA & text & sentiment & pos $\rightarrow$ positive, neg $\rightarrow$ negative \\
\hline
\end{tabular}

Table 9: Optimal task specifications.

After validating candidate task specifications for each task, we have selected the following for conducting our experiments (Table 9).

Providing incorrect or suboptimal specifications to the prompt may cause a large drop in augmentation qualities. For example, in the case of designing task specifications for the COLA dataset, when "linguistic acceptability" is used as the label type (instead of the optimal "grammar"), the downstream performance on the $0.1 \%$ sub-dataset drops to $38.8 \%$, resulting in performance worse than the non-augmented baseline of $68.80 \%$.

\section{RT20 Dataset}

RT20 is a new binary sentiment classification dataset made up of movie reviews posted for movies released in 2020 or thereafter. This newly created dataset is free from the training dataset used by GPT-3, eliminating the possibility of performance improvement due to memorization. 
To build this dataset, we crawled critic reviews of movies released in or after 2020 that were included in the movie category on Rotten Tomatoes ${ }^{8}$. Generally, the critic reviews have higher linguistic acceptability than user reviews, allowing us to control data quality. For each movie, fresh and rotten reviews were sampled at a 1:1 ratio, with "positive" being labeled for fresh reviews and "negative" being labeled for rotten reviews. During preprocessing, all characters were replaced with the lowercase letters, and spaces were added before and after certain special characters: "“.?!:()[],”. The final corpus is a collection of 1,100 positive and 1,100 negative reviews for 62 recent movies. We further split the dataset into 1500 training, 300 validation, and 400 test data using the class-balanced sampling strategy.

\section{GPT3Mix Samples}

The following GPT3Mix examples are generated using the largest GPT-3 model (davinci) on SST-2.

\begin{tabular}{l|l}
\hline Example 1 & $\begin{array}{l}\text { Laughably, irredeemably awful. (negative) } \\
\text { Example 2 } \\
\text { Well-made but mush-hearted. (positive) }\end{array}$ \\
\hline GPT3Mix & Groundbreaking, disturbing. (positive: $75 \%$, negative: $25 \%$ ) \\
\hline \hline Example 1 & $\begin{array}{l}\text { Berry's saucy, full-bodied performance give this aging series a much needed kick, } \\
\text { making "Die Another Day" one of the most entertaining Bonds in years. (positive) } \\
\text { Example 2 } \\
\text { give life to these broken characters who are trying to make their way through } \\
\text { this tragedy. (positive) }\end{array}$ \\
\hline GPT3Mix & $\begin{array}{l}\text { "Gosford Park" takes a leisurely approach to story telling and along the way it mixes } \\
\text { intrigue, comedy, and romance. (positive: } 98 \%, \text { negative: } 2 \%)\end{array}$ \\
\hline \hline Example 1 & $\begin{array}{l}\text { It's just not very smart. (negative) } \\
\text { It's quite an achievement to set and shoot a movie at the Cannes Film Festival } \\
\text { and yet fail to capture its visual appeal or its atmosphere. (negative) }\end{array}$ \\
\hline GPample & $\begin{array}{l}\text { Excessively talky, occasionally absurd and much too long, } \\
\text { Munich is a fascinating mess. (positive: } 21 \%, \text { negative: } 79 \%)\end{array}$ \\
\hline
\end{tabular}

The following examples are generated on CR.

\begin{tabular}{l|l}
\hline Example 1 & $\begin{array}{l}\text { if you are using linux , and you want to use gprs to connect to the net, i suggest this } \\
\text { phone along with a belkin usb bluetooth adapter . (positive) } \\
\text { the eax sound enhancement feature did n 't do enough for me and the custom eq was } \\
\text { a limited 5 band eq . (negative) }\end{array}$ \\
\hline GPT3Mix & $\begin{array}{l}\text { My E550i had 4 months service from Singapore. During those fourmonth I } \\
\text { recognized that this phone is really lovely, not so popular in Indonesia and nobody use } \\
\text { like me :-( Anyway today the Phone set become hot when i try to read message... } \\
\text { (positive: 22\%, negative: 78\%) }\end{array}$ \\
\hline \hline Example 1 & $\begin{array}{l}8 \text { battery life is excellent . (positive) } \\
\text { navigation is a bit tricky and takes getting used to but the included pdf instructions } \\
\text { cover the operation pretty well . (negative) }\end{array}$ \\
\hline GPT3Mix & $\begin{array}{l}\text { Fast and good GPS ! my favorite is the speed camera app sensitivity > ì. <, you } \\
\text { can threshold values ++to avoid spending penalties on shortcuts which detect it many, } \\
\text { i recommend !!! Good product at a great price! (positive: } 99 \%, \text { negative: } 1 \% \text { ) }\end{array}$ \\
\hline
\end{tabular}

The following examples are generated on RT20. Note that GPT-3 would not have learned about some of

\footnotetext{
${ }^{8}$ https://www.rottentomatoes.com/
} 
the movies mentioned in the examples, such as Tenet, hence GPT3Mix cannot retrieve knowledge and facts about the movies. This implies that the data augmentation effect is largely derived from linguistic knowledge and diverse perturbations exhibited by GPT-3. Also note that the parentheses in the second example was in the original review. GPT3Mix learns to mix surface patterns in the examples as well.

\begin{tabular}{|c|c|}
\hline Example 1 & $\begin{array}{l}\text { to be constantly told how tenet's engine works is like having peter jackson explain } \\
\text { the metallurgy behind the one ring - unnecessary . (negative) } \\
\text { an alarmist rise-of-the-machines premise points to a "terminator"-like future, with } \\
\text { very different results, in this serviceable sci-fi war movie . (positive) }\end{array}$ \\
\hline GPT3Mix & $\begin{array}{l}\text { a village blacksmith and a feared highwayman form an alliance to star in the offbeat } \\
\text { arrangement of swords . (positive: } 60 \% \text {, negative: } 40 \% \text { ) }\end{array}$ \\
\hline Exar & $\begin{array}{l}\text { the characters are paper-thin , the plot is super predictable , and despite its star- } \\
\text { studded cast only robert pattinson seems like he's having any fun . (negative) }\end{array}$ \\
\hline Example 2 & $\begin{array}{l}\text { [ there are ] small moments that exude credibility but go hand in hand with others } \\
\text { that don't. (positive) }\end{array}$ \\
\hline GPT3Mix & $\begin{array}{l}\text { [it's ] a robot movie that is fun enough to watch but feels superfluous ; there' s just } \\
\text { not much in here we haven't seen before from superior films like i, robota and } \\
\text { plato 's ashes . (positive: } 10 \% \text {, negative: } 90 \% \text { ) }\end{array}$ \\
\hline Example 1 & $\begin{array}{l}\text { the only thing that makes the film worth watching is a performance by robert } \\
\text { pattinson, who has been the best thing about quite a few films in the last year. } \\
\text { (negative) }\end{array}$ \\
\hline Example 2 & $\begin{array}{l}\text { "land" has real power even when stands absolutely still for lengthy periods of } \\
\text { screen time. (positive) }\end{array}$ \\
\hline GPT3Mix & $\begin{array}{l}\text { the rock will please those who are expecting a visually entertaining. } \\
\text { (positive: } 65 \% \text {, negative: } 35 \% \text { ) }\end{array}$ \\
\hline
\end{tabular}

The following examples are generated on SUBJ. Note that GPT3Mix sometimes struggles to identify the task from the context and predicts the labels with low confidence, as the concept of "subjectivity" can be vague and debatable.

\begin{tabular}{|c|c|}
\hline $\begin{array}{l}\text { Example } 1 \\
\text { Example } 2\end{array}$ & $\begin{array}{l}\text { smith examines the intimate, unguarded moments of folks who live in unusual homes } \\
\text { - which pop up in nearly every corner of the country . (subjective) } \\
\text { this is a film version of the play they wrote based on more than } 200 \text { interviews they } \\
\text { conducted in laramie. (objective) }\end{array}$ \\
\hline GPT3Mix & $\begin{array}{l}\text { reporter covers our corrupt customs laws, and it }{ }^{1} \mathrm{~s} \text { surprising something isn't done } \\
\text { about them . (subjective: } 59 \% \text {, objective: } 41 \% \text { ) }\end{array}$ \\
\hline Example 1 & $\begin{array}{l}\text { " the dangerous lives of altar boys" has flaws, but it also has humor and heart and } \\
\text { very talented young actors (subjective) }\end{array}$ \\
\hline Example 2 & $\begin{array}{l}\text { his family decides to go back on a holiday to india for } 2 \text { weeks, when tina discovers } \\
\text { the truth about pooja's e-mails, they decide together that tina will play along with } \\
\text { the charade. (objective) }\end{array}$ \\
\hline GPT3Mix & $\begin{array}{l}\text { a rich man hires a hitman for his wife . but she finds out and decides to manipulate } \\
\text { the killer with an ever decreasing budget (subjective: } 49 \% \text {, objective: } 51 \% \text { ) }\end{array}$ \\
\hline
\end{tabular}

